\title{
Impact of Infection on the Secretory Capacity of the Male Accessory Glands
}

\author{
M. Marconi, A. Pilatz, F. Wagenlehner, T. Diemer, W. Weidner \\ Department of Urology and Pediatric Urology, University of Giessen, Giessen, Germany
}

\begin{abstract}
Introduction: Studies that compare the impact of different infectious entities of the male reproductive tract (MRT) on the male accessory gland function are controversial.

Materials and Methods: Semen analyses of 71 patients with proven infections of the MRT were compared with the results of 40 healthy non-infected volunteers. Patients were divided into 3 groups according to their diagnosis: chronic prostatitis NIH type II $(\mathrm{n}=38)$, chronic epididymitis $(\mathrm{n}=12)$, and chronic urethritis $(\mathrm{n}=21)$.

Results: The bacteriological analysis revealed 9 different types of microorganisms, considered to be the etiological agents, isolated in different secretions, including: urine, expressed prostatic secretions, semen and urethral smears: E. Coli $(\mathrm{n}=$ 20), Klebsiella $(n=2)$, Proteus spp. $(n=1)$, Enterococcus $(n=20)$, Staphylococcus spp. $(n=1), M$. tuberculosis $(n=2)$, N. gonorrhea $(n=8)$, Chlamydia tr. $(n=16)$ and, Ureaplasma urealyticum $(n=1)$. The infection group had significantly $(\mathrm{p}<0.05)$ lower: semen volume, alpha-glucosidase, fructose, and zinc in seminal plasma and, higher $\mathrm{pH}$ than the control group. None of these parameters was sufficiently accurate in the ROC analysis to discriminate between infected and noninfected men.

Conclusion: Proven bacterial infections of the MRT impact negatively on all the accessory gland function parameters evaluated in semen, suggesting impairment of the secretory capacity of the epididymis, seminal vesicles and prostate. These findings were associated with an infectious related significant increase of semen $\mathrm{pH}$. None of the semen parameters evaluated can be suggested as a diagnostic tool for infection.
\end{abstract}

Key words: accessory sex organs, male; infection; semen; alpha-glucosidases; fructose; zinc

Int Braz J Urol. 2009; 35: 299-309

\section{INTRODUCTION}

Infection of the male reproductive tract (MRT) is a common disease that can deteriorate the quality of spermatozoa and impair the function of the male accessory glands; for this reason it is considered one of the potentially correctable causes of male infertility $(1,2)$. However, the physiopathology and epidemiology regarding the impact of infection on the male accessory gland function is still a matter of debate, and the power of different exocrine function markers of the male accessory glands to discriminate between infected/inflammated vs. non-infected/inflammated patients have been reported with controversial results (3-6).

Male accessory glands secrete several factors such as alpha-glucosidase, fructose, prostaglandins, bicarbonate and citric acid amongst others, which are crucial for sperm physiology. Inflammation "per se" (7) and secondary obstruction (8) have been proposed 
as possible mechanisms through which different infectious agents may impair their function. Under normal conditions the epididymis secretory factors are involved in the maturation of sperm; its function can be evaluated by the measurement of L-carnitine, glycerylphosphoryl choline and alpha-glucosidase in seminal plasma. The secretion of alpha-glucosidase is used to reliably evaluate epididymal function; however, there is no consensus regarding the impact of chronic epididymitis on the level of this marker $(7,9,10)$. The seminal vesicles produce: fructose, ascorbic acid, ergothioneine, prostaglandins and bicarbonate. These factors act as reducing agents and in preventing sperm agglutination (11). A deleterious effect of infection on the secretory function of the seminal vesicles, evaluated by fructose levels has been previously reported (4); however, these findings were not confirmed by other authors $(10,12)$. The secretory function of the prostate gland has been widely investigated: seminal plasma $\mathrm{pH}$, citric acid, gamma-glutamyl transpeptidase and zinc have been proposed as markers of its exocrine function, their concentrations are usually altered in response to bacterial infection and inflammation (1). However, they are currently not recommended as diagnostic tools to detect inflammation or infection in the MRT (5).

Since studies that evaluate the impact of infection on the male accessory gland function still remain controversial, we decided to evaluate the secretory function of the epididymis, seminal vesicles and the prostate, using alpha-glucosidase, fructose and zinc as parameters, in patients with chronic epididymitis, chronic bacterial prostatitis (CBP) and, chronic urethritis.

\section{MATERIALS AND METHODS}

Seventy-one symptomatic consecutive patients (age ranges 23-62) with proven chronic infections of the MRT, attending our special outpatient Department for Urological Andrology were recruited as a study group and 40 age-matched healthy volunteers (age range 20-62), with no previous medical history or evidence of andrological or urological disease and with sterile urine and semen, were enrolled as a control group. The inclusion criteria for the patients enrolled in the study were genital pain or discomfort secondary to infection of the MRT lasting for more than 6 months, no antibiotic uptake for at least six weeks prior the first bacteriological evaluation and, a positive bacteriological finding in the Meares-Stamey 4-glass test. Patients with severe chronic systemic illnesses (i.e. HIV, chemotherapy), previous chronic non-infectious genitourinary diseases under treatment (i.e. benign prostatic hyperplasia under treatment with alpha-blockers or 5 alpha-reductase inhibitors) and, history of prostate biopsy, were excluded from the study. Patients were included in the study irrespective of their fertility status and classified into three groups according to our diagnostic schedule (Table-1).

The diagnosis of CBP was made clinically and based on microbiological tests following the consensus criteria of the NIH $(13,14)$. The diagnosis of chronic epididymitis was performed clinically, sonographically and microbiologically according to consensus statements including search for sexually transmitted disease microorganisms and inflammatory parameters in the ejaculate (peroxidase positive leukocytes, elastase) $(15,16)$. The diagnosis of chronic urethritis was done clinically and microbiologically including the search for sexually transmitted microorganisms and leukocyte counts of the first voided urine and in the ejaculate (peroxidase positive leukocytes, elastase) (16) (Table-1).

\section{Categorization of CBP, Chronic Epididymitis and Chronic Urethritis}

Evaluation of patients with CBP included (17): the NIH chronic prostatitis symptom index German version (18), physical examination including digital rectal examination of the prostate, transrectal ultrasound (TRUS), the 4-glass test with search for common urinary bacteria, mycoplasma and yeasts in all urine fractions (first voided urine: VB1, midstream urine: VB2, and post-prostatic massage urine: VB3) and expressed prostatic secretions (EPS), ejaculate analysis (19), polymerase chain reaction (PCR) for Chlamydia (C.) trachomatis in VB1 (Abbott, Wiesbaden, Germany), and microscopic examination of VB3 for detection of Trichomonas vaginalis (20).

In men with chronic epididymitis, the evaluation included scrotal ultrasound and duplex according 
Table 1 - Laboratory criteria used for categorization of the infectious diseases of the male reproductive tract and bacteriological findings of all patients.

\begin{tabular}{|c|c|c|c|}
\hline & $\begin{array}{l}\text { Prostatitis NIH Type II } \\
\qquad(\mathrm{N}=38)\end{array}$ & $\begin{array}{c}\text { Chronic Epididymitis } \\
\qquad(\mathrm{N}=12)\end{array}$ & $\begin{array}{l}\text { Chronic Urethritis } \\
\qquad(\mathrm{N}=21)\end{array}$ \\
\hline \multirow[t]{2}{*}{ Diagnostic criteria } & $\begin{array}{l}\text { Clinical signs and symptoms } \\
\text { plus typical chronic bacte- } \\
\text { rial prostatitis constellation: } \\
\text { EPS and VB3 > } 10 \text { fold } \\
\text { higher CFU } / \mathrm{mL} \text { than VB1 } \\
\text { and VB2. }\end{array}$ & $\begin{array}{l}\text { Clinical signs and symp- } \\
\text { toms plus either: significant } \\
\text { bacteriuria VB2 }\left(>10^{4}\right. \\
\mathrm{CFU} / \mathrm{mL}), \text { bacteriospermia } \\
\left(>10^{3} \mathrm{CFU} / \mathrm{mL}\right) \text {, and/or } \\
\text { positive } \mathrm{PCR} \text { to } \mathrm{C} \text {. tracho- } \\
\text { matis and/or N. gonorrhea } \\
\text { in VB1. }\end{array}$ & $\begin{array}{l}\text { Clinical signs and symp- } \\
\text { toms plus evidence of } \geq 4 \\
\text { granulocytes per microscopic } \\
\text { field (1000x) in the smear of } \\
\text { urethral discharge, or } \geq 15 \\
\text { granulocytes per microscopic } \\
\text { field ( } 400 \mathrm{x}) \text { in VB1 sediment, } \\
\text { and either: C. trachomatis } \\
\text { or N. gonorrhea positive } \\
\text { PCR in VB1 and/or urethral } \\
\text { smears, or presence of rel- } \\
\text { evant bacteria, mycoplasma, } \\
\text { or yeasts with a concentration } \\
\geq 10^{4} \mathrm{CFU} / \mathrm{mL} \text { in the urethral } \\
\text { smear and/or } \geq 10^{3} \mathrm{CFU} / \mathrm{mL} \\
\text { of } \mathrm{VB} 1 .\end{array}$ \\
\hline & \multicolumn{2}{|c|}{ Bacteriological Findings } & \\
\hline \multicolumn{4}{|l|}{ Aerobic bacteria } \\
\hline Enterococcus & 19 & 1 & - \\
\hline Staphylococcus spp. & 1 & - & - \\
\hline N. gonorrhea & - & - & 8 \\
\hline E. coli & 15 & 5 & - \\
\hline Klebsiella & 2 & - & - \\
\hline M. tuberculosis & - & $2^{1}$ & - \\
\hline \multicolumn{4}{|l|}{ Anaerobic bacteria } \\
\hline C. trachomatis & - & 4 & 12 \\
\hline U. urealyticum & - & - & $1^{2}$ \\
\hline
\end{tabular}

${ }^{1}$ History of epididymal tuberculosis.

${ }^{2}>10^{3} \mathrm{CFU} / \mathrm{mL}$ of $U$. urealyticum in $\mathrm{VBI}$

to consensus suggestions for evaluation of patients with epididymitis (15). The microbiological evaluation included PCR in VB1 for C. trachomatis and Neisseria (N.) gonorrhea, and search for common relevant bacteria in VB2 and in the ejaculate (16) (Table-1).

In patients with chronic urethritis, microbiological evaluation included microscopic evaluation of VB1 with cytological analysis for leukocytes (Papanicolaou-staining), semi quantitative culture methods for relevant bacteria $(\mathrm{N}$. gonorrhea, mycoplasmas and Candida spp.) in urethral discharge and in VB1, and PCR for C. trachomatis and N. gonorrhea (Abbott, Wiesbaden, Germany) in urethral smears and in VB1. Evidence of $\geq 4$ granulocytes per microscopic field (X1000) in the urethral discharge smear, or $\geq 15$ granulocytes per microscopic field (X400) in VB1 sediment, and either: $\mathrm{C}$. trachomatis or N. gonorrhea positive PCR in VB1 and/or urethral smears, or presence of common bacteria, mycoplasmas, or yeasts 
with a concentration $\geq 10^{4} \mathrm{CFU} / \mathrm{mL}$ in the urethral discharge and/or $\geq 10^{3} \mathrm{CFU} / \mathrm{mL}$ in VB1, were considered criteria for diagnosis (16) (Table-1).

\section{Detection of Microorganisms}

The bacteriological analysis of the patients revealed 9 different types of infectious agents isolated in different MRT secretions including urine, EPS, urethral smears and semen (Table-1). The most common isolated microorganisms in all patients were Escherichia (E.) coli $(\mathrm{n}=20)$ and Enterococcus spp. $(n=20)$. When the different diseases were analyzed separately, Enterococcus spp. was the most common agent isolated in patients with CBP and, in patients with chronic epididymitis, E. coli was the most prevalent. Infections due to sexually transmitted microorganisms were only detected in men suffering from chronic epididymitis and chronic urethritis. Two patients had a previous history of epididymitis due to Mycobacterium (M.) tuberculosis previously treated with antibiotics.

\section{Ejaculate Analysis}

Complete ejaculate analysis according to the WHO standards (19) including semen volume, $\mathrm{pH}$, elastase and peroxidase positive leukocytes (PPL) determination was performed in all men (21). Levels of $\alpha$-glucosidase and fructose (total enzymatic activity) at neutral $\mathrm{pH}$ were determined by spectrophotometrical methods described elsewhere (5). Zinc was assessed using a commercially available kit (Zinc Kit, Bako, Germany).

The impact of inflammation on the levels of semen volume, $\mathrm{pH}, \alpha$-glucosidase, fructose and zinc was analyzed stratifying the patients as having an inflammatory or non-inflammatory spermiogram according to two well accepted criteria: PPL $\geq 1 \times 10^{6} / \mathrm{mL}$ (19) and/or elastase $\geq 230 \mathrm{ng} / \mathrm{mL}$ (21).

\section{Statistical Analysis}

Data were analyzed by the Prisma program for Windows version 5.0. Mann-Whitney U, KruskalWallis and Dunn's multiple comparison test were used to analyze the results of the ejaculate and, Receiver
Operating Characteristic Curves (ROCC-analysis) was applied to assess the normal ranges of the seminal plasma parameters in the cases where statistical difference was found. Statistical significance was achieved at $\mathrm{p}<0.05$, all reported $\mathrm{p}$ values are two-sided.

\section{RESULTS}

\section{Cytomorphological Analysis of the Ejaculate}

Compared with the controls, the patients had statistically significantly $(p<0.05)$ lower sperm concentration, $\%$ of sperm with progressive motility $(\mathrm{a}+\mathrm{b}), \%$ of sperm with normal morphology; and higher: $\%$ of immotile sperm, $\%$ of sperm with head deformity, and $\%$ of sperm with tail deformity. No significant differences were observed in the levels of PPL, elastase and sperm vitality between the two groups (Table-2).

\section{Secretory Parameters of the Male Accessory Glands}

Compared to the controls, the patients had statistically significantly $(\mathrm{p}<0.05)$ lower (mean \pm $\mathrm{SD})$ : semen volume $(4.1 \pm 1.5$ vs. $2.6 \pm 1.8 \mathrm{~mL})$, levels of $\alpha$-glucosidase (79.1 \pm 56.1 vs. $54.6 \pm 46.8$ $\mathrm{mU} /$ ejaculate), levels of fructose ( $59.5 \pm 40.4 \mathrm{vs} .38 .1$ $\pm 34.2 \mu \mathrm{mol} /$ ejaculate $)$, levels of zinc $(24.1 \pm 18.4 \mathrm{vs}$. $12.1 \pm 14.1 \mathrm{U} /$ ejaculate $)$ and, higher $\mathrm{pH}(8.1 \pm 0.4$ vs. $8.3 \pm 0.4)$ (Table-3).

We applied a ROCC analysis to quantify a cut point to discriminate men with versus without infection using the parameters of male accessory gland function that were significantly different in the infection group $(\mathrm{n}=71)$. A semen volume of 2.75 $\mathrm{mL}$ was found to discriminate patients from controls with a sensitivity of $62.9 \%$ and specificity of $90.9 \%$, the Area Under the Curve (AUC) was 0.77, a level $\alpha$-glucosidase of $56.1 \mathrm{mU} /$ ejaculate discriminated men with versus without infection with a sensitivity of $73 \%$ and specificity of $60 \%$ (AUC: 0.65 ), a level of fructose of $47.5 \mu \mathrm{mol} / \mathrm{ejaculate}$ discriminated men with versus without infection with a sensitivity of $73 \%$ and specificity of $60 \%$ (AUC: 0.68 ) and, a level of zinc of 14.2 U/ejaculate discriminated men with 
Table 2 - Physical and cytomorphological analysis of the ejaculate in controls $(N=40)$ and patients $(N=71)$.

\begin{tabular}{lcc}
\hline Semen Analysis Parameter & $\begin{array}{c}\text { Control Group } \\
\text { Mean } \pm \text { SD }\end{array}$ & $\begin{array}{c}\text { Infection Group } \\
\text { Mean } \pm \text { SD }\end{array}$ \\
\hline $\begin{array}{l}\text { Sperm count (mio/mL) } \\
\text { Agglutination }(0-+++)\end{array}$ & $142 \pm 100.9$ & $97.4 \pm 147^{*}$ \\
Vitality eosin test \% & 0 & + \\
Motility & $81.5 \pm 8.8$ & $78.2 \pm 17.6$ \\
$\quad$ Progressive motility \% (a+b) & $62.2 \pm 14.9$ & $50.5 \pm 19^{*}$ \\
$\quad$ Nonprogressive motility \% (c) & $17.4 \pm 6.2$ & $9.5 \pm 6.1^{*}$ \\
$\quad$ Immotile sperm \% (d) & $20.4 \pm 13.1$ & $39.0 \pm 17.6^{*}$ \\
Morphology & & $7.6 \pm 7.6^{*}$ \\
$\quad$ Normal \% & $39.4 \pm 12.4$ & $72.8 \pm 15.5^{*}$ \\
$\quad$ Head deformity \% & $29.4 \pm 9.7$ & $27.1 \pm 17.8$ \\
$\quad$ Midpiece deformity \% & $21.2 \pm 8.9$ & $26.3 \pm 14.0^{*}$ \\
$\quad$ Tail deformity \% & $36.7 \pm 15.4$ & \\
Cell count & & \\
$\quad$ Peroxidase positive (mio/mL) & $0.1 \pm 0.17$ & \\
Biochemical analysis & & $305.7 \pm 328.5$ \\
$\quad$ Elastase (ng/mL) & $176.4 \pm 114.1$ & \\
\hline
\end{tabular}

*Significant statistical difference in the control group ( $p<0.05$, Mann-Whitney U test).

versus without infection with a sensitivity of $75.4 \%$ and specificity of $70 \%$ (AUC: 0.79 ). A semen $\mathrm{pH}$ of 8.15 was found to discriminate patients from controls with a sensitivity of $58.8 \%$ and specificity of $63.6 \%$ (AUC: 0.67) (Figure-1).

The multiple comparison analysis between the different diagnoses, microorganisms and control group, revealed that patients with CBP had statistically significant $(\mathrm{p}<0.05)$ lower: semen volume
$(4.1 \pm 1.5$ vs. $2.5 \pm 1.7 \mathrm{~mL})$, levels of fructose $(59.5$ \pm 40.4 vs. $37.2 \pm 32.9 \mu \mathrm{mol} /$ ejaculate), and levels of zinc ( $24.1 \pm 18.4$ vs. $10.9 \pm 13.7$ U/ejaculate $)$ than the controls. Patients with chronic urethritis had statistically significant $(\mathrm{p}<0.05)$ lower: semen volume $(4.1$ \pm 1.5 vs. $2.1 \pm 1.1 \mathrm{~mL})$ and levels of zinc $(24.1 \pm 18.4$ vs. $13.7 \pm 15.1 \mathrm{U} /$ ejaculate) than the controls. Patients with chronic epididymitis had statistically significant $(\mathrm{p}<0.05)$ lower levels of zinc $(24.1 \pm 18.4 \mathrm{vs} .13 .1 \pm$

Table 3 - Comparison of the different secretory parameters of the male accessory glands between the controls and patients.

\begin{tabular}{lcc}
\hline Biochemical Analysis & $\begin{array}{c}\text { Control Group } \\
\text { Mean } \pm \text { SD }\end{array}$ & $\begin{array}{c}\text { Infection Group } \\
\text { Mean } \pm \text { SD }\end{array}$ \\
\hline Volume $(\mathrm{mL})$ & $4.1 \pm 1.5$ & $2.6 \pm 1.8^{*}$ \\
pH & $8.1 \pm 0.4$ & $8.3 \pm 0.4^{*}$ \\
Alpha-glucosidase (mU/ejaculate) & $79.1 \pm 56.1$ & $54.6 \pm 46.8^{*}$ \\
Fructose $(\mu \mathrm{mol} /$ ejaculate) & $59.5 \pm 40.4$ & $38.1 \pm 34.2^{*}$ \\
Zinc $(\mathrm{U} /$ ejaculate) & $24.1 \pm 18.4$ & $12.1 \pm 14.1^{*}$ \\
\hline
\end{tabular}

*Significant statistical difference in the control group $(p<0.05$, Mann-Whitney U test). 


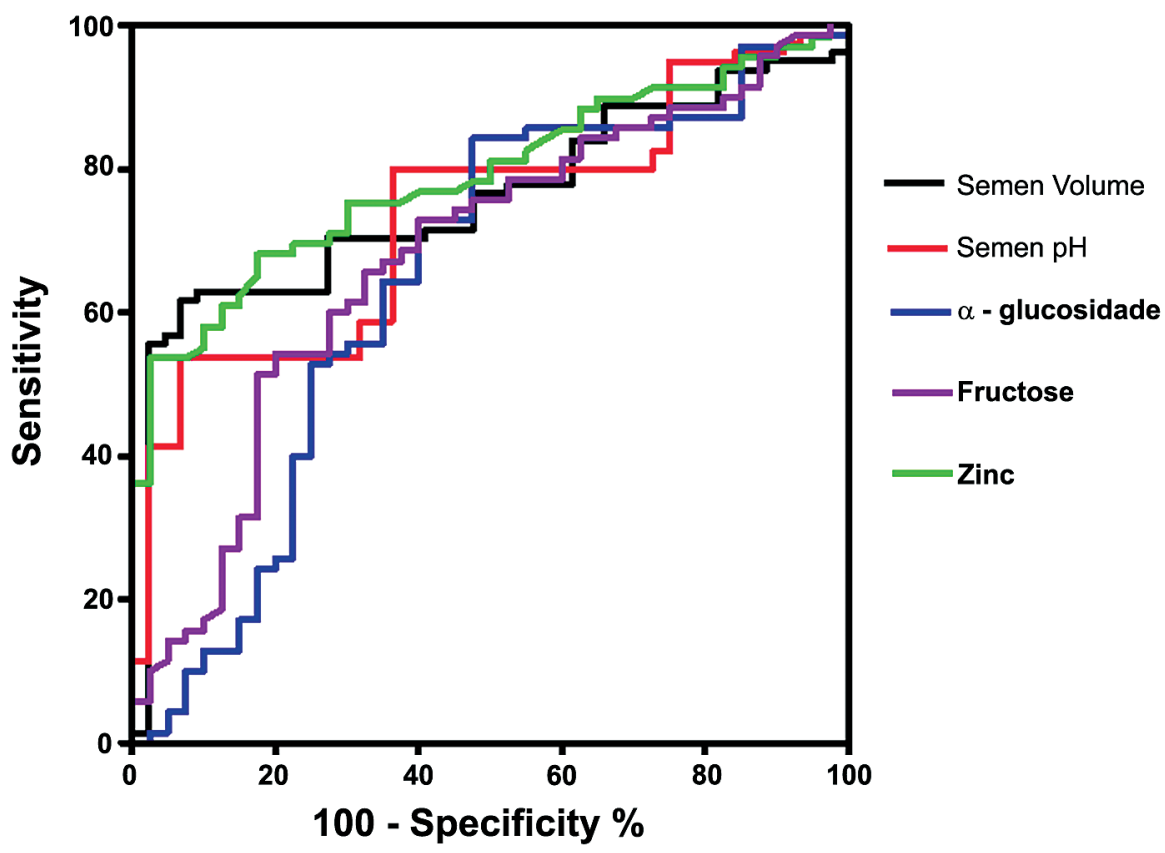

Figure 1-ROCC analysis of semen volume, semen $\mathrm{pH}$, alpha-glucosidase, fructose and zinc levels, to differentiate men with vs. without MRT infection. Semen volume $=$ cut point $2.75 \mathrm{~mL}$; sensitivity $=62.9 \%$; specificity $=90.9 \%$; AUC $=0.77$. Semen pH=cut point 8.15 ; sensitivity $=58.8 \%$, specificity $=63.6 \%$, AUC $=0.67$. Alpha-glucosidase: cut point 14.2 mU/ejaculate, sensitivity $=73 \%$, specificity $=$ $65 \%$; $A U C=0.65$. Fructose $=$ cut point 47.5 umol/ejaculate; sensitivity $=73.0 \%$; specificity $=60.0 \% ;$ AUC $=0.68$. Zinc $=$ cut point 14.2 U/ejaculate, sensitivity $=75.4 \%$; specificity $=70.0 \% ; A U C=0.79$.

14.2 U/ejaculate) than the controls. Patients infected with E. coli had statistically significant $(p<0.05)$ lower levels of fructose $(59.5 \pm 40.4$ vs. $23.9 \pm 18.4$ $\mu \mathrm{mol} /$ ejaculate), lower levels of zinc ( $24.6 \pm 18.4 \mathrm{vs}$. $6.3 \pm 7.2 \mathrm{U} /$ ejaculate), reduced semen volume (4.1 \pm 1.5 vs. $2.2 \pm 1.4 \mathrm{~mL})$, and higher $\mathrm{pH}(8.1 \pm 0.4$ vs. $8.5 \pm 0.4)$ compared to the control group. Patients infected with $\mathrm{N}$. gonorrhea presented statistically significant $(\mathrm{p}<0.05)$ lower semen volume $(4.1 \pm 1.5$ vs. $1.8 \pm 1.2 \mathrm{~mL})$ and lower levels of zinc $(24.6 \pm 18.4$ vs. $8.0 \pm 8.5 \mathrm{U} /$ ejaculate) than the control group. No other significant differences between the patients and the controls were observed considering all the other specific diagnoses and microorganisms evaluated.

The subgroup of patients with inflammatory signs in the spermiogram according to two criteria: $\mathrm{PPL} \geq 1 \times 10^{6} / \mathrm{mL}$ (19) and/or elastase $\geq 230 \mathrm{ng} / \mathrm{mL}$ (21) $(n=36)$, demonstrated also a significant decrease in the levels of semen volume, alpha-glucosidase, fructose and zinc compared to the controls and, higher values of semen $\mathrm{pH}$ (data not shown). The sensitivity and specificity of these parameters to diagnose MRT infection in patients with inflammatory signs in the ejaculate were; semen volume: cut point $2.75 \mathrm{~mL}$, sensitivity $63.2 \%$, specificity $90.9 \%$, AUC 0.79 ; semen $\mathrm{pH}$ : cut point 8.15 , sensitivity $61.1 \%$, specificity $63.6 \%$, AUC 0.70; alpha-glucosidase cut point 56.1 $\mathrm{mU} /$ ejaculate, sensitivity of $73 \%$ specificity of $60 \%$, AUC: 0.65 ; fructose: cut point: $47.5 \mu \mathrm{mol} /$ ejaculate, sensitivity of $73 \%$, specificity of $60 \%$, AUC: 0.68 ; zinc: cut point $10.7 \mathrm{U} /$ ejaculate, sensitivity $83 \%$, specificity $80 \%$, AUC 0.88 .

\section{COMMENTS}

In the clinical setting MRT infection has a questionable effect on the fertility prognosis. What seems to be clear is that the different factors secreted by the male accessory glands are crucial for normal conception, since sperm retrieved directly from the epididymis or the testis are unable to fertilize the 
egg without the assistance of artificial reproduction techniques (22). Taking this fact into account, it is interesting to analyze the effect of different infectious entities of MRT and their etiologic agents on the secretory function of the male accessory glands. Mainly two questions need to be answered, first if the different diseases and microorganisms can cause obstruction of the seminal pathways and by that means deteriorate the normal composition of the ejaculate; and second if they are related to any intrinsic impairment of the secretory function of the epididymis, seminal vesicles and prostate.

The fact that all patients included in the study group had proven MRT infectious diseases defined according to well accepted consensus definitions, allowed us to accurately identify the origin of the MRT infection. Moreover, in previous reports semen cultures identify significant bacteriospermia in only $50 \%$ of semen specimens from men with CBP, thus ejaculate culture is not yet recommended for the first line of diagnostic management in men with suspected CBP (23). The microbiological findings in our study group are in agreement with previous reports (1); N. gonorrhea and $\mathrm{C}$. trachomatis are the most frequent infectious agents isolated patients with chronic urethritis. In patients with CBP, Enterococcus and E. coli were, as expected, the more frequent microbiological agents isolated. Finally, it is interesting that in patients consulting for chronic epididymitis, the microbiological findings gave similar results to those found in cases of acute epididymitis (15), suggesting that under special conditions the epididymis may act as a reservoir of bacteria in the male reproductive tract.

In our series of patients, we did not find any azoospermic patients, although, the levels of alphaglucosidase, fructose, zinc and sperm concentration were significantly lower in the infection group compared to the control group. Also, there were no conclusive findings indicating a total obstruction of the MRT at any level. This fact confirms that obstruction is not an important cause of impairment of the male accessory gland function in an infectious setting and although infection has been previously mentioned in the literature as common cause of obstruction of the MRT (2), our findings and more recent studies $(6,20)$ seem to confirm that it is a rare occurrence in patients with demonstrated MRT infection and inflammation.
Analyzing the secretory function of the epididymis we found that men with MRT infectious diseases $(n=71)$ had significantly lower concentration of $\alpha$-glucosidase. However, in the multivariate analysis no significant differences were found in the levels of this marker between any specific diagnosis and the controls, even in patients with chronic epididymitis. Moreover, no specific bacteria had a significant impact on its level. Although Cooper and co-workers (10) found a significant decrease in the level of alphaglucosidase in patients with acute epididymitis; in patients with chronic epididymitis previous reports suggest that the impact on secretory function of the epididymis is not significant (9). We propose that our series of patients reflect that the secretion of alpha-glucosidase is significantly decreased in cases of chronic infections of the MRT; however, this decrease is not as pronounced compared to the decrease observed in the levels of others markers, i.e. zinc.

The function of the seminal vesicles can be accurately determined by the level of fructose in seminal plasma, questionable since these glands produce the majority of the semen volume, this parameter may also be used as indirect indicator of its function. When discussing the literature, the impact of infection on the secretory function of the seminal vesicles remains controversial. Comhaire and coworkers (4), in agreement with our results, found a negative impact of infection on the fructose level, but concluded that the measurement of its concentration was not useful for discriminating between infected and non-infected patients due to its low sensitivity and specificity. Vicari and co-workers (24) demonstrated that patients with prostate-vesiculo-epididymitis had significantly lower seminal fructose levels than those patients with prostatitis alone. On the other hand, Bezold and co-workers (12) did not find a significant decrease in the concentration of fructose in seminal plasma in a population of infertile men with sexually transmitted diseases. The controversial results regarding the impact of MRT infection on fructose levels may be explained by the different clinical and microbiological criteria used to include patients in the studies, we believe that the evaluation of patients with suspected MRT infection should follow consensus criteria and guidelines e.g. NIH criteria for patients with prostatitis $(13,14)$ or the European guidelines for the 
management of epididymo-orchitis for patients with epididymitis (15). Using these diagnostic procedures and criteria to manage our patients, we found a negative impact of infection for the MRT on the fructose level. In contrast, if we had considered the fact that the seminal vesicles produce approximately $80 \%$ of the ejaculate volume, a decrease in this parameter would indicate at least in part, that the secretory functions of these glands were impaired, most probably secondary to an intrinsic damage rather than to obstruction, since azoospermia was not found in any patient indicating a normal passage of seminal fluid to the colliculus. The negative effect of infection and inflammation on semen volume has been previously described $(25,26)$. Although semen volume was significantly lower in our patients, the low sensitivity and specificity in the ROCC analysis prevents us from suggesting it as a diagnostic tool to detect MRT infection.

Secretory dysfunction of the prostate gland is a common finding in patients with documented prostatitis. Our previously reported research (5) found, that the levels of gamma-GT were significantly decreased in patients with inflammatory chronic prostatitis/chronic pelvic pain syndrome (CP/CPPS); however, it cannot be recommended as a diagnostic tool for inflammation due to its low sensitivity and specificity. In this new study group, including only patients with documented infection, the levels of zinc, identified as prostatic secretory markers were significantly lower. These findings agree with previous reports demonstrating lower levels of zinc in the seminal plasma of patients with MRT infection (27). However, again the power of this marker to discriminate infected from non-infected patients (sensitivity $43.6 \%$, specificity $75 \%$ ) was low.

Although, a zinc-rich diet can slightly increase the concentration of this element in the prostate gland (28); there is no conclusive evidence that this therapy can increase the zinc concentration in semen, making the rationale of this therapy unclear.

Semen $\mathrm{pH}$ is determined by the acid secretions of the prostate and the alkaline secretions of the seminal vesicles. In vitro, external $\mathrm{pH}$ is an important factor in the regulation of sperm physiology. An acid $\mathrm{pH}$ contributes to maintain a non-capacitated state preventing premature acrosomal reaction (29). Higher levels of semen $\mathrm{pH}$ in patients with MRT infection have been reported in the literature and could reflect, at least in part, a secretory dysfunction of the prostate due to lower levels of citric acid in semen (30). The importance of semen $\mathrm{pH}$ in fertility prognosis of these patients is not clear. However, from a diagnostic point of view semen $\mathrm{pH}$ can not be recommended as a tool to discriminate infected from non-infected patients, due to its low sensitivity and specificity.

Nevertheless, all the evaluated parameters of the secretory function of the accessory glands were significantly altered in patients with concomitant infection and inflammation of the MRT $(n=36)$ compared to controls. In the ROCC analysis the sensitivity and specificity of these factors to detect infection was not significantly increased, when compared to the whole infection group analysis $(n=71)$ and were not significant when inflammatory activity was considered.

Finally, a constellation that includes inflammatory signs in the spermiogram (PPL $\geq 1 \times 10^{6} / \mathrm{mL}$ and/or elastase $\geq 230 \mathrm{ng} / \mathrm{mL}$ ), of low semen volume, elevated semen $\mathrm{pH}$ and low levels of alpha-glucosidase, fructose and zinc could indicate the presence of an infection; however, the sensitivity and specificity of these parameters, prevents their application as a diagnostic tool for the detection of MRT infection.

\section{CONCLUSIONS}

Infection of the male reproductive tract significantly decreases the levels of semen volume, alpha-glucosidase, fructose and zinc in seminal plasma suggesting impairment of the secretory function of the epididymis, seminal vesicles and prostate. Due to their low sensitivity and specificity these parameters can not be recommended as a diagnostic tool to detect MRT infection. Although semen $\mathrm{pH}$ is significantly increased in patients with infection of the male reproductive tract, its sensitivity and specificity to detect infection are low.

\section{ACKNOWLEDGEMENTS}

M. Marconi, M.D. is a fellow in "Clinical Andrology" and received a scholarship from MIDEPLAN, Chile. 


\section{CONFLICT OF INTEREST}

\author{
None declared.
}

\section{REFERENCES}

1. Weidner W, Krause W, Ludwig M: Relevance of male accessory gland infection for subsequent fertility with special focus on prostatitis. Hum Reprod Update. 1999; 5: 421-32.

2. World Health Organization: WHO Manual for the Standardized Investigation, Diagnosis and Management of the Infertile Male. Cambridge University Press. 2000; 2nd Ed.

3. Eggert-Kruse W, Zwick EM, Batschulat K, Rohr G, Armbruster FP, Petzoldt D, et al.: Are zinc levels in seminal plasma associated with seminal leukocytes and other determinants of semen quality? Fertil Steril. 2002; 77: 260-9.

4. Comhaire FH, Vermeulen L, Pieters O: Study of the accuracy of physical and biochemical markers in semen to detect infectious dysfunction of the accessory sex glands. J Androl. 1989; 10: 50-3.

5. Ludwig M, Vidal A, Diemer T, Pabst W, Failing K, Weidner W: Seminal secretory capacity of the male accessory sex glands in chronic pelvic pain syndrome (CPPS)/chronic prostatitis with special focus on the new prostatitis classification. Eur Urol. 2002; 42: 24-8.

6. Weidner W, Wagenlehner FM, Marconi M, Pilatz A, Pantke KH, Diemer T: Acute bacterial prostatitis and chronic prostatitis/chronic pelvic pain syndrome: andrological implications. Andrologia. 2008; 40: 105-12.

7. Mahmoud AM, Geslevich J, Kint J, Depuydt C, Huysse L, Zalata A, et al.: Seminal plasma alpha-glucosidase activity and male infertility. Hum Reprod. 1998; 13: 591-5.

8. Dohle GR: Inflammatory-associated obstructions of the male reproductive tract. Andrologia. 2003; 35: 321-4.

9. Wolff H, Bezold G, Zebhauser M, Meurer M: Impact of clinically silent inflammation on male genital tract organs as reflected by biochemical markers in semen. J Androl. 1991; 12: 331-4.

10. Cooper TG, Weidner W, Nieschlag E: The influence of inflammation of the human male genital tract on secretion of the seminal markers alpha-glucosidase, glycerophosphocholine, carnitine, fructose and citric acid. Int J Androl. 1990; 13: 329-36.

11. Okamura N, Tajima Y, Ishikawa H, Yoshii S, Koiso $\mathrm{K}$, Sugita Y: Lowered levels of bicarbonate in seminal plasma cause the poor sperm motility in human infertile patients. Fertil Steril. 1986; 45: 265-72.

12. Bezold G, Politch JA, Kiviat NB, Kuypers JM, Wolff $\mathrm{H}$, Anderson DJ: Prevalence of sexually transmissible pathogens in semen from asymptomatic male infertility patients with and without leukocytospermia. Fertil Steril. 2007; 87: 1087-97.

13. Krieger JN, Nyberg L Jr, Nickel JC: NIH consensus definition and classification of prostatitis. JAMA. 1999; 282: 236-7.

14. Schaeffer AJ, Anderson RU, Krieger JN, Lobel B, Naber K, Nakagawa M, Nickel JC, Nyberg L, Weidner $\mathrm{W}$ : The Assessment And Management Of Male Pelvic Pain Syndrome Including Prostatitis. In: Mc Connel J, Abrams P, Denis L, Khoury S, Roehrsom C (eds.), Male Lower Urinary Tract Dysfunction. Evaluation and Management6th International Consultation in Prostate Cancer and Prostate Diseases. Health Publications, Paris. 2006; pp. 343-385.

15. Horner PJ; European Branch of the International Union against Sexually Transmitted Infection and the European Office of the World Health Organization: European guideline for the management of epididymo-orchitis and syndromic management of acute scrotal swelling. Int J STD AIDS. 2001; (12 Suppl 3): 88-93.

16. Schiefer HG: Microbiology of male urethroadnexitis: diagnostic procedures and criteria for aetiologic classification. Andrologia. 1998; (30 Suppl 1): 7-13.

17. Weidner W, Anderson RU: Evaluation of acute and chronic bacterial prostatitis and diagnostic management of chronic prostatitis/chronic pelvic pain syndrome with special reference to infection/inflammation. Int J Antimicrob Agents. 2008; (31 Suppl 1): S91-5.

18. Schneider H, Brähler E, Ludwig M, Hochreiter W, Collins MF, Eremenco S, et al.: Two-year experience with the German-translated version of the NIH-CPSI in patients with CP/CPPS. Urology. 2004; 63: 102730 .

19. World Health Organization Laboratory Manual for the Examination of Human Semen and Semen-Cervical Mucus Interaction. Cambridge University Press, Cambridge. 1999; 4th ed.

20. Ludwig M, Vidal A, Diemer T, Pabst W, Failing K, Weidner W: Chronic prostatitis/chronic pelvic pain pyndrome): seminal markers of inflammation. World J Urol. 2003; 21: 82-5.

21. Ludwig M, Kümmel C, Schroeder-Printzen I, Ringert $\mathrm{RH}$, Weidner W: Evaluation of seminal plasma parameters in patients with chronic prostatitis or leukocytospermia. Andrologia. 1998; (30 Suppl 1): 41-7. 
22. Diemer T, Schroeder-Printzen I, Weidner W: Surgical sperm retrieval. Urologe A. 2007; 46: 789-98.

23. Weidner W, Wagenlehner FM, Marconi M, Pilatz A, Pantke KH, Diemer T: Acute bacterial prostatitis and chronic prostatitis/chronic pelvic pain syndrome: andrological implications. Andrologia. 2008; 40: 10512.

24. Vicari E, La Vignera S, Castiglione R, Calogero AE: Sperm parameter abnormalities, low seminal fructose and reactive oxygen species overproduction do not discriminate patients with unilateral or bilateral postinfectious inflammatory prostato-vesiculo-epididymitis. J Endocrinol Invest. 2006; 29: 18-25.

25. Sanocka-Maciejewska D, Ciupinska M, Kurpisz M: Bacterial infection and semen quality. J Reprod Immunol. 2005; 67: 51-6.

26. Engeler DS, Hauri D, John H: Impact of prostatitis NIH IIIB (prostatodynia) on ejaculate parameters. Eur Urol. 2003; 44: 546-8.
27. Wang Y, Kang L, Hou Y, Wu X, Chen J, Han X: Microelements in seminal plasma of infertile men infected with Ureaplasma urealyticum. Biol Trace Elem Res. 2005; 105: 11-8.

28. Cortesi M, Fridman E, Volkov A, Shilstein SSh, Chechik R, Breskin A, et al.: Clinical assessment of the cancer diagnostic value of prostatic zinc: a comprehensive needle-biopsy study. Prostate. 2008; 68: 994-1006.

29. Parrish JJ, Susko-Parrish JL, First NL: Capacitation of bovine sperm by heparin: inhibitory effect of glucose and role of intracellular pH. Biol Reprod. 1989; 41: 683-99.

30. Comhaire FH, Vermeulen L, Pieters O: Study of the accuracy of physical and biochemical markers in semen to detect infectious dysfunction of the accessory sex glands. J Androl. 1989; 10: 50-3.
Accepted after revision: January 20, 2009

\section{Correspondence address:}

Dr. Wolfgang Weidner

Dept of Urology \& Pediatric Urology

Justus-Liebig-Universität Giessen

Rudolf-Buchheim-Strasse 7

Giessen, 35385, Germany

Fax: + 490641 99-44509

E-mail: wolfgang.weidner@chiru.med.uni-giessen.de

\section{EDITORIAL COMMENT}

Male accessory gland infection (MAGI) is a syndrome which includes clinical symptoms of inflammation of the prostate gland, the seminal vesicles, the ductus deferens and the epididymis. There is not a clear discrimination against the term prostatis, glandulitis vesicalis or epididymitis. There is also not a clear classification of MAGI as formulated for prostatitis, although it is likely that different types of MAGI in terms of infectious and non-infectious causes also exist.
Usually MAGI is caused by infectious agents spreading from the urethra via prostate gland, seminal vesicles, ductus deferens, and epididymis. The frequency of a changeover from urethritis to MAGI is unknown. Infections by viral agents are to date hypothetical.

MAGI may lead to obstruction of the ductus epididymidis, impairment of spermatogenesis in epididymo-orchitis, to impairment of sperm function and to the induction of sperm auto-antibodies, as 
well as to dysfunctions of the male accessory glands, which leads to decreased seminal concentrations of citric acid, phosphatase, fructose concentration, zinc and alpha-glutamyltransferase.

In general, however, the impact of MAGI on semen composition and sperm function, possibly relevant for male fertility, is low and vice versa, none of the dysfunctions can be considered suitable for the diagnosis of MAGI. The paper clearly shows that the diagnosis of MAGI on the basis of semen analysis is difficult and that the diagnosis of prostatitis and epididymitis is more reliably performed by means of an examination of prostatic fluid and imaging procedures.

Dr. Walter Krause

Dept. Dermatology \& Allergology University Hospital, Philipp University

Marburg, Germany

E-mail:krause@med.uni-marburg.de 